\title{
Piecewise nonlinear goal-directed portfolio insurance strategies under TIPP idea
}

\author{
Jiah-Shing Chen ${ }^{1}$ Benjamin Penyang Liao ${ }^{1,2}$ \\ ${ }^{1}$ Department of Information Management, National Central University, \\ Chungli 320, Taiwan \\ ${ }^{2}$ Department of Information Management, Overseas Chinese Institute of Technology, \\ Taichung 407, Taiwan
}

\begin{abstract}
Traditional portfolio insurance (PI) strategy, such as CPPI, only considers the floor constraint but not the goal aspect. This paper proposes a goal-directed (GD) strategy to express an investor's goal-directed trading behavior and combines this floor-less GD strategy with the goal-less CPPI strategy to form a piecewise linear goal-directed CPPI (GDCPPI) strategy. In addition, we extend the piecewise linear GDCPPI strategy to be a piecewise nonlinear GDCPPI strategy. Furthermore, we replace CPPI concept by TIPP idea to generate related GDTIPP strategies. The piecewise GDCPPI and GDTIPP strategies are special cases of goaldirected portfolio insurance (GDPI) strategy. This paper applies genetic algorithm (GA) technique to find better piecewise linear GDPI strategy parameters than those under the Brownian motion assumption. This paper also applies forest genetic programming (GP) technique to generate the piecewise nonlinear GDPI strategy. The statistical tests show that the GP strategy outperforms the GA strategy which in turn outperforms the Brownian strategy.
\end{abstract}

Keywords: Portfolio insurance strategy, goal-directed strategy, piecewise GDCPPI strategy, piecewise GDTIPP strategy.

\section{Trading Strategies}

\subsection{Portfolio insurance strategy}

Suppose an investor tries to maximize the growth rate of expected utility of the final wealth under the portfolio insurance constraint [6]. The problem becomes:

$$
\begin{gathered}
\sup _{X} \lim _{T \rightarrow \infty} \frac{1}{\gamma T} \ln E\left[\gamma U\left(W_{T}\right)\right] \\
\text { subject to } W_{t} \geq F, \forall t \leq T,
\end{gathered}
$$

where $X$ denotes the set of admissible trading strategies, $W_{T}$ is the final wealth, $W_{t}$ is the wealth at period $t, 0 \neq \gamma \leq 1$, and $F>0$ is the floor. If $F$ is fixed, the optimal strategy to the above optimization problem is:

$$
x_{t}=\frac{\mu}{\sigma^{2}(1-\gamma)}\left(W_{t}-F\right),
$$

where $x_{t}$ is the amount dollars invested into the risky asset, $\mu$ and $\sigma$ are the mean and standard deviation of return rates. Eq. (2) can be simplified as:

$$
\zeta_{t} \equiv x_{t}=m_{1}\left(W_{t}-F\right), W_{t} \geq F
$$

where $m_{1}=\frac{\mu}{\sigma^{2}(1-\gamma)}$ can be regarded as the investor's risk multiplier, $F$ is the floor. This $\zeta_{t}$ is the popular CPPI strategy, which is a special case of portfolio insurance (PI) strategy.

Most PI strategies consider only the floor and are therefore goal-less. An investor will have a greater chance of failing his almost reached goal when the current wealth is very high.

\subsection{Risk attitudes}

Evidences show that an investor will change his riskattitude under different wealth levels. In particular, studies showed that fund managers change their riskattitudes based on their performance compared to the benchmark. However, there are contradictory observations among these studies. Some studies $[8,4]$ observed that fund managers take risk-averse behavior when their performance is worse than the benchmark (low wealth risk aversion) while other studies [1, 7] observed that fund managers take risk-seeking behavior when their performance is worse than the benchmark (high wealth risk aversion). These two types of risk-attitude are described as follows.

Low wealth risk aversion: An investor will become risk-averse when his current wealth is low and will become risk-seeking when his current wealth is high.

High wealth risk aversion: An investor will become risk-averse when his current wealth is high and will become risk-seeking when his current wealth is low. 
The goal-less CPPI strategies demonstrate the low wealth risk aversion phenomenon. Goal-directed perspective proposes that an investor in financial markets will consider certain investment goal. A goal-directed investor will take risk-seeking behavior when the distance from current wealth to the goal (goal distance) is large and will take risk-averse behavior when the goal distance is small.

\subsection{Goal-directed strategy}

In the study by Browne [2], one of the investment problems is to maximize the survival probability in danger zone or to maximize the probability of reaching the goal before reaching the bankruptcy point. The model can be described as follows.

$$
\min _{X} P\left(\tau_{a}>\tau_{b}\right) \text {, s.t. } a<W_{t}<b<S,
$$

where $X$ is the set of admissible strategies, $P(\cdot)$ is the probability function, $a$ is the bankruptcy point, $\tau_{a}$ is the escape time when $W_{t}=a, \tau_{b}$ is the escape time when $W_{t}=b, S$ is the safe point and is generally set up to be $c / r$, with $c$ being the minimal consumption, and $r$ being the risk-free rate of return. This model tries to find an optimal trading strategy which minimizes the probability of reaching the bankruptcy point $a$ before reaching the goal $b$. The optimal strategy turns out to be:

$$
x_{t}=\frac{2 r}{\mu-r}\left(S-W_{t}\right),
$$

where $\mu$ is the mean of return rates for the risky asset. If $b \rightarrow S, S$ in fact can be regarded as the goal $G$ that an investor wants to achieve. Then we define a goaldirected $(G D)$ strategy as

$$
\eta_{t} \equiv x_{t}=m_{2}\left(G-W_{t}\right), W_{t} \leq G,
$$

where $m_{2}=\frac{2 r}{\mu-r}$ is a constant.

The GD strategy shows that an investor should take a riskier action when goal distance is large and should take less riskier activity when goal distance is small. This behavior is consistent with the high wealth risk aversion.

\subsection{Piecewise linear goal-directed CPPI strategy}

Intuitively, investors will take different strategy when they posit different risk attitude. That is, if their risk attitude is low wealth risk aversion, they will adopt CPPI strategy. If their risk attitude is high wealth risk aversion, they will adopt GD strategy.

A new problem with combined constraint $F \leq$ $W_{t} \leq G$ from CPPI and GD strategies then can be derived. At first we develop a combined piecewise linear GDCPPI strategy [3], which is defined as: $\pi_{t} \equiv$ $x_{t}=c\left[\lambda\left(W_{t}-F\right)+(1-\lambda)\left(G-W_{t}\right)\right], 0 \leq \lambda \leq$
$1, F \leq W_{t} \leq G$. However, this strategy can not hold the full features of CPPI and GD strategies under extreme wealth values (e.g., $W_{t}=F$ or $W_{t}=G$ ). In further analysis, there is a wealth position $M$ projected from the intersection of these CPPI and GD strategies. The value of $M$ can be calculated by

$$
M=\frac{m_{1} F+m_{2} G}{m_{1}+m_{2}} .
$$

Since CPPI strategy only considers the floor $F$ but not the goal $G$, an investor can apply CPPI strategy when $W_{t}<M$. On the other hand, GD strategy only considers the goal $G$ but not the floor $F$, an investor can apply GD strategy when $W_{t} \geq M$. We therefore build a piecewise linear goal-directed CPPI (GDCPPI) strategy as:

$$
\theta_{t} \equiv x_{t}=\left\{\begin{array}{l}
0, W_{t} \leq F \\
m_{1}\left(W_{t}-F\right), F<W_{t}<M \\
m_{2}\left(G-W_{t}\right), M \leq W_{t} \leq G
\end{array}\right.
$$

Note that $\theta_{t}$ is a generalization of both CPPI and GD strategies. In particular, if $m_{1} \rightarrow \infty, M=$ $\frac{m_{1} F+m_{2} G}{m_{1}+m_{2}}=F$ and the constraint $M \leq W_{t} \leq G$ for GD segment will be $F \leq W_{t} \leq G$. Therefore, piecewise linear GDCPPI strategy reduces to GD strategy. If $m_{2} \rightarrow \infty, M=\frac{m_{1} F+m_{2} G}{m_{1}+m_{2}}=G$ and the constraint $F \leq W_{t}<M$ for CPPI segment will be $F \leq W_{t}<G$. Therefore, piecewise linear GDCPPI strategy reduces to CPPI strategy.

In addition, Eq. (8) can be generalized to be:

$$
\theta_{t} \equiv x_{t}=\min \left(\zeta_{t}, \eta_{t}\right)
$$

This generalized strategy can cause the $M$ value to be implicit.

Traditional CPPI strategy is based on the assumption of Brownian motion for stock prices. Browne's study [2] for goal seeking objective also made this assumption. When investors try to apply these above strategies, the parameter values are generally obtained by the long-term expectation method. That is, the mean and variance of return rates are the long-term expectations from historical data.

However, the historical data might not follow the Brownian motion. Better parameter values for $m_{1}$ and $m_{2}$ in piecewise linear GDCPPI strategy might be directly obtained using other data driven optimization methods with historical data. Genetic algorithm is the method chosen to search better parameters values for $m_{1}$ and $m_{2}$ in this study due to its success in many applications.

\subsection{Piecewise nonlinear goal-directed CPPI strategy}

The piecewise linear GDCPPI strategy is based on the assumption of linearity. However, the sub-strategies within the framework of piecewise strategy need not 
to be linear when such as the Brownian motion assumption does not hold anymore. The piecewise linear GDCPPI strategy can then logically be extended to a piecewise nonlinear GDCPPI strategy by relaxing the linear strategy structure to the nonlinear one and defined as:

$$
\theta_{t}^{\prime} \equiv x_{t}=\min \left(\zeta_{t}^{\prime}, \eta_{t}^{\prime}\right)
$$

where $\zeta_{t}^{\prime}=\aleph\left(W_{t}, F\right)$ is a nonlinear CIPP-Oriented strategy, $\aleph(\cdot)$ is a nonlinear function, and $\eta_{t}^{\prime}=$ $\aleph\left(W_{t}, G\right)$ is a nonlinear GD strategy.

\subsection{Applying TIPP idea}

Time invariant portfolio protection (TIPP) idea proposes the floor is dynamic according to the current wealth [5]. The TIPP strategy tries to protect investment returns when pursuing maximal performance. In this study, we apply the TIPP concept to replaces the floor $F$ in CPPI with $\alpha K_{t}$, where $K_{t}=\max _{\tau \leq t} W_{\tau}$ is the maximum wealth up to time $t$ and $\alpha \in(0,1)$ is a constant. The TIPP version of the GD strategy is obtained by simply replacing the goal $G$ with $\beta K_{t}$, where $\beta>1$ is a constant. We then define a piecewise linear goal-directed TIPP (GDTIPP) strategy as:

$$
\psi_{t} \equiv x_{t}=\min \left(\xi_{t}, \delta_{t}\right),
$$

where $\xi_{t}$ is the TIPP strategy and $\delta_{t}$ is a linear GD strategy with TIPP idea. In addition, we define a piecewise nonlinear GDTIPP strategy as:

$$
\psi_{t}^{\prime} \equiv x_{t}=\min \left(\xi_{t}^{\prime}, \delta_{t}^{\prime}\right)
$$

where $\xi_{t}^{\prime}=\aleph\left(W_{t}, K_{t}, \alpha\right)$ is a nonlinear TIPPOriented strategy, $\aleph(\cdot)$ is a nonlinear function, and $\delta_{t}^{\prime}=\aleph\left(W_{t}, K_{t}, \beta\right)$ is a nonlinear GD strategy with TIPP idea. The piecewise GDCPPI and GDTIPP strategies are then special cases of goal-directed portfolio insurance (GDPI) strategy.

\section{Experiments and Analyses}

The main experimental purpose in this study tries to justify our proposition for piecewise GDPI strategies: there are piecewise nonlinear GDPI strategies can outperform piecewise linear GDPI strategies. Three experiments, B-GP, GA-GP, and B-GA experiments, are then executed to show that GP strategy (generated by GP) can outperform both B and GA strategies (generated by Brownian and GA techniques), and GA strategy can outperform B strategy. The ROI will be applied as the performance measure.

Some parameter values are derived by three pretests. The first pretest tries to decide a suitable pair of year length and $\gamma$ values for B technique, where $\gamma$ is defined in Eq. (1). The year length is decided to calculate the expected values of return rate $\mu$ and variation $\sigma^{2}$. In turn, the $\mu, \sigma^{2}$ and $\gamma$ will be used to calculate the parameters $m_{1}$ and $m_{2}$ in piecewise linear GDPI strategy for B technique, where $m_{1}$ is defined in Eq. (3) and $m_{2}$ is defined in Eq. (6). The pretest shows that the year length is 8 and $\gamma$ is 0.1 . The second pretest tries to decide the learning length for GA learning. The third pretest tries to decide the learning length for GP learning. Both the second and third pretests show that the better learning length is 100 trading days.

We randomly select 5 stocks as the experimental targets from the 30 components of Dow Jones Industrial Average (DJIA), namely, American International Group (AIG), IBM, Merck (MRK), HP (HPQ), and Exxon Mobil (XOM). We also randomly select 5 starting learning dates, which are 1999/12/13, 2001/6/6, 2002/2/27, 2003/4/28 and 2004/12/03. Three different starting floors in the experiments for piecewise strategies are pre-assigned and calculated by the ratios of floor to initial wealth, which are $0.7,0.8$ and 0.9 , and applied as $\alpha$ values in TIPP case. Also 3 different starting goals in the experiments for piecewise strategies are pre-assigned and calculated by the ratios of goal to initial wealth, which are 1.1, 1.2 and 1.3, and applied as $\beta$ values in TIPP case. The risk-free rate of return is 0.0001 per day. For each sub-experiment, there are $(5 * 5 * 3 * 3=) 225$ cases and then generates 225 samples for statistical tests. For each sub-experiment, the testing length is 30 days.

\subsection{GA learning}

For GA learning, parameter values $m_{1}$ and $m_{2}$ will be optimized. These two parameters are both encoded into 14-bit long strings and decoded within [1.0, 13.7]. The fitness function for each experiment is to maximize the ROI. The other main GA system parameters are set up as follows: the population size is 40; each run executes 20 generations and then there are 800 fitness evaluations in each run; crossover is twopoint; mutation rate is 0.001 per bit; and the selection method is integral roulette wheel selection. The searching space then will be $2^{14}=4096$ points and we in fact search 800 points which is about $25 \%$ searching space.

\subsection{Forest genetic programming}

A piecewise strategy consists of multiple substrategies. Each sub-strategy has its own objective and application condition. When applying traditional GP to generate a piecewise strategy, each sub-strategy can be encoded as a tree and the whole piecewise strategy is represented as a forest. Adapting a single forest chromosome implies the evolution of individual trees. We then generalize traditional GP into a forest GP for dealing with the piecewise characteristics.

Each tree in forest GP can have its own objective, function set and terminal set, which can be totally independent from other tree's. Therefore, the framework of forest GP can be designed to process the multiobjective problem. However, in order to show the basic 
features of piecewise strategy, we reduce the multiple objectives into a single objective framework by designating each sub-strategy having the same objective.

\subsection{Forest GP learning}

In this forest GP learning, better piecewise nonlinear GDPI strategies will be generated. In forest GP, terminal set, function set and other system parameters are pre-assigned as follows. For PI strategy, the terminal set for forest GP includes floor, current wealth, and constant within $[-127,127]$. The function set includes operators $+,-, *, /$. For GD strategy, the terminal set for forest GP includes goal, current wealth, and constant within $[-127,127]$. The function set includes operators $+,-, *, /$.

The fitness function for each experiment is to maximize the ROI. The GP population size is 40 . GP runs 200 generations. The depth is 4 , which the depth of root node is 1 .

\subsection{Design for statistical tests}

Three types of paired-samples $t$ tests are applied to check the advantages among B, GA, and GP strategies for ROI. The first type of tests tries to show the GA strategy can outperform the B strategy. The second type of tests tries to show the GP strategy can outperform the GA strategy. The third type of tests tries to show the GP strategy can outperform the B strategy. There are totally 6 paired-samples $t$ tests will be processed.

\subsection{Results of statistical tests}

The results of 6 paired-samples $t$ tests for out sample data are shown in Table 1.

Table 1: Paired-samples $t$ tests for out samples of piecewise GDCPPI and GDTIPP strategies.

\begin{tabular}{|l|c|c|}
\hline pair & t-value & Sig. \\
\hline$R O I_{B}(\theta)-R O I_{G A}(\theta)$ & -2.303 & $.0110^{* *}$ \\
\hline$R O I_{G A}(\theta)-R O I_{G P}\left(\theta^{\prime}\right)$ & -3.622 & $.0000^{* * *}$ \\
\hline$R O I_{B}(\theta)-R O I_{G P}\left(\theta^{\prime}\right)$ & -4.046 & $.0000^{* * *}$ \\
\hline$R O I_{B}(\psi)-R O I_{G A}(\psi)$ & -3.278 & $.0005^{* * *}$ \\
\hline$R O I_{G A}(\psi)-R O I_{G P}\left(\psi^{\prime}\right)$ & -2.604 & $.0050^{* * *}$ \\
\hline$R O I_{B}(\psi)-R O I_{G P}\left(\psi^{\prime}\right)$ & -3.103 & $.0010^{* * *}$ \\
\hline \multicolumn{2}{|l|}{$d f=224,{ }^{* * *}: p<0.01,{ }^{* *}: p<0.05$} \\
\hline
\end{tabular}

We can see that pair tests of out sample of B-GA, GA-GP and B-GP for both GDCPPI and GDTIPP strategies all reach the statistical significance. These experimental results can justify that there are piecewise nonlinear GDPI strategies can outperform the piecewise linear GDPI strategies at ROI measure with statistical significance.

A selected case of experimental piecewise nonlinear GDTIPP strategy, $\psi_{t}^{\prime}=\min \left(\xi_{t}^{\prime}, \delta_{t}^{\prime}\right)$, for stock MRK, starting testing date: $2002 / 7 / 22$, is shown as following:

$$
\left\{\begin{array}{l}
\xi_{t}^{\prime}=W_{t}-(-128)-(-128), \\
\delta_{t}^{\prime}=(G / 33) /\left(W_{t}-100\right) .
\end{array}\right.
$$

The generated GP strategy does show the nonlinear characteristics which are different from $\mathrm{B}$ and GA strategies.

\section{Conclusions}

Under considering the goal constraint, this paper firstly develops a goal-directed strategy which is different from the traditional portfolio insurance strategy. We combine the concepts of goal-seeking and portfolio insurance to form piecewise linear and nonlinear goaldirected portfolio insurance strategies under CPPI concept and TIPP idea. In order to justify our propositions, we make some experiments and the outputs support them with statistical significance.

The characteristics of forest genetic programming will be exploited further. The piecewise trading strategy is interesting and is worthy to develop further in the future.

\section{References}

[1] K. Brown, W. Harlow, and L. Starks, "Of tournaments and temptations: An analysis of managerial incentives in mutual fund industry," Journal of Finance, 51, pp. 85-110, 1996.

[2] Sid Browne, "Survival and growth with a liability: Optimal portfolio strategies in continuous time," Mathematics of Operations Research, 22(2), pp. 468-493, 1997.

[3] J. S. Chen and B. P. Y. Liao, "Goal-directed portfolio insurance," ICNC 2005, LNCS 3612, pp. 798807, 2005.

[4] J. Chevalier and G. Ellison, "Risk taking by mutual funds as a response to incentives," Journal of Political Economy, 105, pp. 1167-1200, 1997.

[5] T. Estep and M. Kritzman, "TIPP: Insurance without complexity," Journal of Portfolio Management, 14, pp. 38-42, 1988.

[6] S. J. Grossman and Z. Zhou, "Optimal investment strategies for controlling drawdowns," Mathematical Finance, 3(3), pp. 241-276, 1993.

[7] J. C. Jackwerth, "Recovering risk aversion from option prices and realized returns," The Review of Financial Studies, 13(2), pp. 433-451, 2000.

[8] J. Tayler, "Risk-taking behavior in mutual fund tournaments," Journal of Economic Behavior \& Organization, 50, pp. 373-383, 2003. 